East African Medical Journal Vol. 77 No. 6 June 2000

BRAIN ABSCESS AT THE KENYATTA NATIONAL HOSPITAL, NAIROBI

N. J. M. Mwang'ombe, MMed (Surg), PhD (Lond), Department of Surgery, College of Health Sciences, University of Nairobi, P.O. Box 19676, Nairobi, Kenya.

\title{
BRAIN ABSCESS AT THE KENYATTA NATIONAL HOSPITAL, NAIROBI
}

\author{
N. J. M. MWANG'OMBE
}

\begin{abstract}
Objective: To study the aetiology, mode of presentation and outcome following treatment of brain abscesses at the Kenyatta National Hospital, Nairobi.

Design: A retrospective study.

Setting: Kenyatta National Hospital, Nairobi.

Subjects: Sixty five patients with brain abscesses who were seen at Kenyatta National Hospital between January 1989 and December 1993.

Results: Twenty patients died following surgery (30.7\% mortality). Eight per cent of the patients who underwent surgery were serologically positive for the human immune deficiency virus (HIV). When these patients were excluded mortality was $25 \%$. There were more male patients than females (ratio 2.4:1). Thirty eight per cent of the patients were children below the age of ten years. Trauma was the commonest cause of brain abscess. The aetiology was unknown in $24 \%$ of the cases. Twenty six patients who were HIV positive and had suspected brain abscesses were not included in this study because they did not undergo surgery. The commonest organisms isolated wereStaphylococci, Streptococci, Klebsiella and Haemophylus influenzae. Sixty eight per cent of the patients had seizures. All the patients were diagnosed by computerised tomography (CT) scanning of the brain. Surgical treatment was by multiple burr hole aspirations and excision through craniotomy.

Conclusion: Brain abscess is still a major cause of morbidity at the Kenyatta National Hospital with a high overall mortality of $30.7 \%$ during the period under study.
\end{abstract}

\section{INTRODUCTION}

A brain abscess consists of localised free or encapsulated pus within the brain substance. Abscesses may vary in size from a microscopic focus of inflammatory cells to a large encapsulated area of necrosis occupying a major part of the cerebral hemisphere. They may be single or multiple and caused by local extension or haematogenous spread(1). Brain abscess is about one sixth as frequent as bacterial meningitis. There is a male predominance of 2:1(1). The most common sites are frontal and temporal lobes(1). Approximately $30 \%$ of all brain abscesses occur in children less than 15 years of age with a peak incidence between ages four and seven years(1). The course of the illness may range from indolent to fulminant. The duration of symptoms in two thirds of the patients may be two weeks or less. Clinical manifestations are due to the space occupying mass. Computerised tomograhy (CT) scanning with intravenous contrast is necessary to assist in the diagnosis of abscess(2). The contrast media demonstrates capsular enhancement commonly referred to as "ring enhancement". Magnetic resonance imaging (MRI) is an excellent diagnostic procedure if available(1). Surgery is the definite treatment for cerebral abscess. Spread of infection is minimised by administering antibiotics. The availability of CT scanning has allowed a reduction in mortality of from 30-40 to about 10\%(3).
In this study, clinical records of patients treated for brain abscess at the Kenyatta National Hospital Nairobi over a five year period were reviewed to determine the clinical pattern, treatment and outcome following treatment.

\section{MATERIALS AND METHODS}

Over a period of five years from January 1989 to December 1993, sixty five patients were treated for brain abscesses at the Kenyatta National Hospital. The files of these patients were retrospectively reviewed with respect to age at presentation, aetiology, location of the abscess, sex distribution, human immune deficiency virus (HIV) status of the patients and out-come following treatment.

\section{RESULTS}

Sixty five patients with brain abscesses were treated at the Kenyatta National Hospital between January 1989 and December 1993 (Figure 1). An average of 13 cases were seen per year. Their age ranged between four months and seventy years (Figure 2). Males were more common than females (ratio of 2:4:1). Twenty five per cent of the patients seen were below the age of five years and $38 \%$ of the patients were below the age of 10 years (Figure 2). Trauma was the commonest cause of brain abscess (35\%). Other common causes were leptomeningitis and cranial osteomyelitis (21\%), mastoiditis, middle ear infection and sinus infection (20\%) 
(Table 1). Cases of brain abscesses arising from spread from a distant foci such as a lung infection were not common in this study $(6 \%)$. The most common site for brain abscess was the parietal lobe (54\%). Most of the abscesses found in the parietal lobe were due to penetrating trauma. The next common site was the temporal lobe (17\%) and most of these cases were secondary to otogenic or mastoid infections. Five patients $(8 \%)$ who tested positive for the human immunodeficiency virus (HIV) underwent surgery. All these patients died following surgery. Twenty six patients who tested positive for HIV and had suspected brain abscess were not included in this study because they did not undergo surgery for one reason or the other. The aetiology was unknown in nine per cent of the cases. There was a delay of more than 76 hours before surgery on head injury patients with depressed compound fractures awaiting surgery who subsequently developed brain abscess. Twenty patients died while undergoing treatment following surgery $(30.7 \%$ mortality). Five of these were HIV positive. When these were excluded mortality was $23 \%$. Mortality from trauma related abscesses was $18 \%$ and HIV associated abscess, $100 \%$. Seizures were present in $68 \%$ of the patients. Cultures were positive in forty eight per cent of the patients, and most of the cultures had two or more organisms. The commonest organisms were Staphylococci, Streptococci, Klebsiella and Haemophylus influenzae. Cultures obtained from HIV positive patients were negative. Surgical treatment consisted of burr hole aspiration or excision through craniotomy.

\section{Figure 1}

Total number of cases of brain abscesses seen between 1989-1993 65 patients: average, 13 patients per year

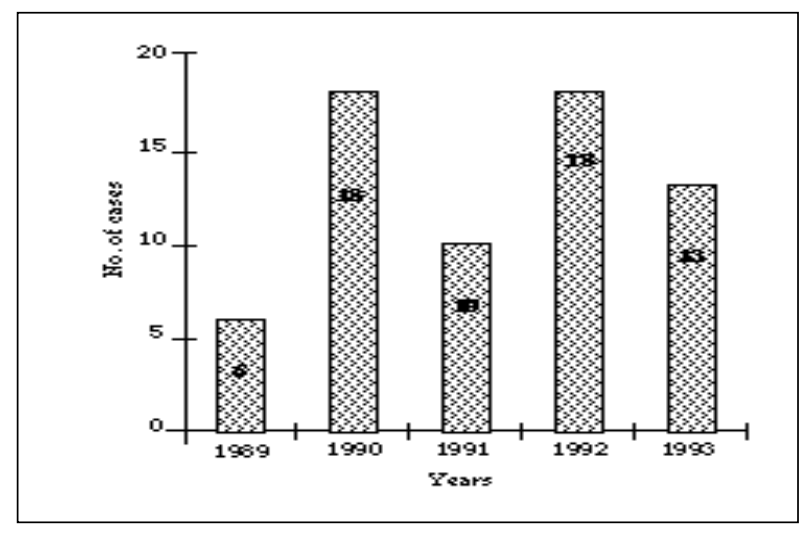

Table 1

Causes of brain abscess at Kenyatta National Hospital, 1989-1993

\begin{tabular}{lr}
\hline Cause & Frequency \\
\hline Penetrating trauma/post craniotomy & $23(35 \%)$ \\
Mastoiditis, middle ear disease, sinus infection & $13(20 \%)$ \\
Leptomeninigitis, cranial osteomyelitis & $14(21 \%)$ \\
Metastatic from lung infection & $4(6 \%)$ \\
Human immune deficiency virus-associated & $5(8 \%)$ \\
Not clear & $6(9 \%)$ \\
Died & $20(30.7 \%$ mortality) \\
& $25 \%$ excluding HIV +ve)
\end{tabular}

Figure 2

Age distribution

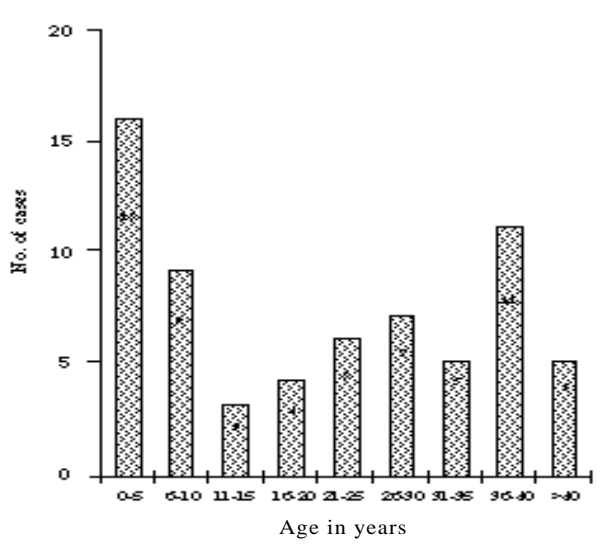

All the patients had CT scanning of the brain. Ring enhancement was common after contrast injection. Where possible, CT scanning was utilised in the management of patients undergoing repeated aspirations.

\section{DISCUSSION}

A previous study done at the Kenyatta National Hospital which reviewed cases of brain abscess seen between 1970 and 1979 found that four patients with brain abscess were seen per year(4). This study was done at a period when the CT scan was not available in Nairobi. Carotid and vertebral angiography was then the main diagnostic test. A brain abscess appears as an avascular mass in carotid angiography. Nowadays CT scanning is the best diagnostic procedure available in patients believed to have a brain abscess. Ring enhancement may be seen in $90 \%$ of cases. In this study, an average of thirteen patients with brain abscesses were seen per year between 1989 and 1993 at the Kenyatta National Hospital, Nairobi. This increase in the number of cases may be due to the improved diagnostic facilities available in Kenya or to the overall increase in population in the country and Nairobi in particular. Another factor which may have contributed to an increase in incidence of brain abscesses at the Kenyatta National Hospital is the human immune-deficiency virus which accounted for $7.7 \%$ of surgically treated cases seen in this study.

Twenty five per cent of the patients seen in this study were under five years of age. These findings are similar to those of Ndirangu seen in the same institution between 1970 and 1979(4).

The large number of male patients seen in this study (M:F: 2.4:1) is probably related to the high incidence of trauma related cases of brain abscesses.

The most common cause of brain abscesses in this study was trauma related causes (compound fractures, bullet wounds, intracranial foreign bodies) which accounted for thirty five percent of all the cases seen. This was most likely related to delay in carrying out definitive surgery in head injury patients leading to subsequent development of brain abscess, a common problem at Kenyatta National Hospital. In most cases, these delays were related to the 
fact that these patients were usually referred from other health facilities for specialised care.

In the developed world brain abscess accounts for approximately one in 10,000 general hospital admissions(5). The incidence of brain abscess varies with geographic location and socio-economic conditions. In developed countries a busy neurosurgical service may encounter two to four patients with brain abscess per year. The incidence of brain abscess is much higher in the developing world(5). The overall incidence of brain abscess worldwide has decreased since the advent of antibiotic therapy. However, the incidence of brain abscess in the developing world remains high.

Brain abscesses remain associated with significant mortality and morbidity. The increase in the numbers of immune compromised patients in recent years has impacted heavily on the incidence of abscesses caused by opportunistic infections(6). The organisms that cause brain abscess may arrive in the brain by direct extension, by haematogenous dissemination or by direct trauma. In upto one third of patients the origin remains unknown(5). In this study the aetiology was not clear in nine per cent of the patients.

Bacteria from an infected area are spread transdurally by direct extension or through the venous channels. Spread of a middle ear and mastoid infection may result in a temporal lobe abscess or, less likely, a cerebellar abscess. A paranasal sinus infection may result in a frontal lobe abscess(5).

The haematogenous spread of bacteria may result in one or more brain abscesses. Common origins for haematogenous dissemination include a dental infection, a pulmonary infection (lung abscess, empyema, pneumonia) a cardiac source (intracardiac shunt, endocarditis), a skin pustule, diverticulitis of the bowel and osteomyelitis. The majority of haematogenously disseminated lesions occur in the frontal and parietal lobes(5).

A brain abscess may be the result of direct trauma such as an open skull fracture or penetrating injury, or it may follow a surgical procedure. The incidence of abscess following an open skull fracture in the developed world is 3 to $7 \%$. The incidence of abscess is higher in the developing world(5). The incidence in this study was found to be $35 \%$.

Prior to the antibiotic era Staphylococcus aureus was a common pathogen in brain abscesses, along with aerobic streptococci. With aggressive antibiotic treatment of serious staphylococcal infections, anaerobic bacteria are now the most common cause of most brain abscesses. In immunecompetent patients mixed anaerobes and aerobes predominate, andPeptostreptococcus, Bacteroides species and Haemophylus influenzae are common organisms $(7,8)$. Immune compromised patients are at risk for fungal abscesses caused by Candida albicans and Aspergillus species(5). Infants can develop abscesses after meningitis which are caused by Proteus and citro bacteria species(9). Antibiotic treatment initiated before culture results are available should include broad spectrum agents and metronidazole. Post-operative antibiotic therapy is continued for six to eight weeks(5).

Although conservative management with high dose antibiotics has been successfully used with small lesions, the mainstay of abscess management is surgical treatment for both decompression of mass lesions and the identification of the pathogen in order to optimise antibiotic coverage(5). Both open surgical excision and aspiration are frequently used. In cases of retained foreign material, such as penetrating trauma, excision is preferred. Multiple deep lesions are often amenable to CT or MRI guided stereotactic drainage(10).

Excision offers the advantage of removing infected necrotic tissue and may be associated with a lower recurrence rate. Excision is ideal when a thick capsule has formed or for solitary abscesses in accessible locations. Excision may not be ideal in patients in poor medical condition. Aspiration can be performed stereotactically, freehand or under ultra sound guidance through a burr hole or craniotomy(5).

Due to the high incidence of seizures, patients with intracerebral abscess should be on anticonvulsant therapy for a minimal of six months(5). In this study $68 \%$ of the patients had seizures. Eight per cent of the patients with brain abscesses who underwent surgery in this study were HIV-positive and had no other obvious factors which could have contributed to the aetiology. However, twenty six patients with suspected brain abscesses seen during this period who tested positive for HIV did not undergo surgery due to their poor general condition. These patients were not included in this study.

The overall mortality at Kenyatta National Hospital remains high at $30.7 \%$. Similar results were observed in the previous study by Ndirangu in the same institution(4). However, when the HIV positive cases were excluded, the mortality was twenty three percent. These figures are still high when compared with those reported from the developed world in the CT scan era $(10 \%)(3)$.

\section{ACKNOWLEDGEMENTS}

We gratefully acknowledge the cooperation and assistance of the Director, Kenyatta National Hospital and the Research and Ethics Committee, Kenyatta National Hospital, for permission to carry out this study and publish these findings.

\section{REFERENCES}

1. Selby R., Ramirez G. B., Sigh R., Kleopouslos I., Kusne S., Starzl T.E. and Fung J. Brain abscess in solid organ transplant recipients receiving cyclosporine-based immunosuppression. Arch. Surg. 1997; 132: 304 310.

2. Salzman C. and Tuazon C.U. Value of the ring-enhancing sign in differentiating intra-cerebral haematomas and brain abscesses. Arch. Intern. Med. 1987; 147

3. Miller E.S., Dias P.S. and Uttley D. CT scanning in the management of intra cranial abscess. A review of 100 cases. Brit. J. Neurosurg. 1988; 2:439-46.

4. Ndirangu K. A study of brain abscesses 1970 - 1979. MMed Thesis University of Nairobi, 1980.

5. Osenback R.K. and Loftus C.M.: "Diagnosis and Management of Brain Abscess", in Neurosurgery Clinics of North America: Infections in Neurological Surgery, Haines S.J. and Hall W.A. (Eds.), 1992, 3(2), $403-420$.

6. Pons V.G., Jacobs R.A. and Hollander M. Non viral infections of the Central Nervous System. Raven Press, New York, 1991.

7. Dolan R.W. and Chowdhury K. Diagnosis and treatment of intra cranial complications of paranasal sinus infections. J. Oral and Maxillofac. Surg. 1995; 5:1080 - 7 .

8. Singh B., Van Dellen J., Ramjettan SN. and Maharaj T.J. Sinogenic intra cranial complications. J. Laryngol. Otol. 1995; 109:945 - 50.

9. Graham D.R. Citrobacter diversus brain abscess and meningitis in neonates. J. Amer. Med. Ass. 1981; 245: 1923 - 25.

10. Dyste G.N., Hitchon P.W. and Menezes A.N. Stereotaxic surgery in the treatment of multiple brain abscesses. J. Neurosurg. 1988;9: 188 -194. 